\title{
Acoustic orbital angular momentum Hall effect and realization using a metasurface
}

\author{
Xu-Dong Fan and Likun Zhang ๑* \\ Department of Physics and Astronomy, National Center for Physical Acoustics, University of Mississippi, University, Mississippi 38677, USA
}

(Received 29 June 2020; revised 29 September 2020; accepted 22 January 2021; published 18 March 2021)

\begin{abstract}
Vortex beams with a twisted wavefront possess both intrinsic and extrinsic orbital angular momenta. Their coupling (viz. orbital-orbital coupling) leads to an orbital angular momentum Hall effect previously found in light, providing applications in light manipulation. Here an analogous orbital Hall effect in acoustics is reported via modeling vortex beam propagation in an inhomogeneous medium. A shift of the beam center perpendicular to the inhomogeneous direction is numerically observed to follow a path predicted by ray theory in the weak inhomogeneity approximation where the coupling and conservation of total angular momenta are identified. Experimental observation of the acoustic phenomenon with the aid of a gradient metasurface or a stratified fluid is proposed.
\end{abstract}

DOI: 10.1103/PhysRevResearch.3.013251

\section{INTRODUCTION}

Interactions between different types of angular momenta possessed by optical waves lead to a Hall effect of light [1-7], which is an analog of the spin Hall effect in electronic systems [8-10] and provides promising applications in precision manipulations of light and photonics devices [11-13]. Specially, optical waves possess spin angular momenta (SAM) by circular polarization, intrinsic orbital angular momenta (IOAM) by an azimuthal phase gradient, and extrinsic orbital angular momenta (EOAM) related to the motion of the beam center. The coupling between SAM and EOAM influences the polarization and the trajectory of the beam center, causing: (i) a trajectory-dependent polarization variation characterized by the Berry phase, providing a parallel transport of the polarization vector along the ray, and (ii) the spin Hall effect of light, which leads to splitting of rays for different polarizations in a smoothly inhomogeneous medium or the transverse Imbert-Fedorov shifts in reflection and refraction of light at a sharp boundary [14-16]. Apart from the spin Hall effect, there also exist the orbital angular momentum Hall effect in light, caused by the coupling between IOAM and EOAM [4,1721]. A spin-orbit Hall effect is also found recently for optical waves [7].

Although the Hall effect and topological phenomena in optics have been studied for decades, the angular momentum Hall effect in acoustics has not been reported in the past decades of acoustic vortex beam studies [22-30]. The study on topological acoustics just started a few years ago [31-49], and the Berry geometric phase effect in acoustics was not reported until recently $[45,50]$. The propagation of acoustic vortex

\footnotetext{
*zhang@olemiss.edu
}

Published by the American Physical Society under the terms of the Creative Commons Attribution 4.0 International license. Further distribution of this work must maintain attribution to the author(s) and the published article's title, journal citation, and DOI. beams in an inhomogeneous media was simulated recently [51] where the fundamental phenomenon of the Hall effect in acoustics is still missing.

Here, we report a numerical observation and theoretical characterization of the orbital angular momentum Hall effect related to the coupling between IOAM and EOAM in acoustics. We observe the transverse shifts of the beam center related to the orbital Hall effect via simulating and modeling a vortex beam propagating in a smoothly inhomogeneous medium. The simulated results are compared with a theoretical prediction characterizing the dependence of the shift on physical parameters (helicity, wave number, and medium inhomogeneity). Our paper reveals the existence of the orbital Hall effect in an acoustic setting and opens up the study of angular momentum coupling in acoustics. Experiments using gradient metasurfaces or stratified fluids are proposed to observe the phenomenon.

\section{NUMERICAL OBSERVATION OF THE HELICITY-DEPENDENT TRANSVERSE SHIFT}

We simulate an acoustic vortex beam propagating in a linearly stratified medium by using a commercial software COMSOL MULTIPHYSICS (ACOUSTIC MODULE). The dimensions of the computational domain are set to $\lambda_{0} \times 3 \lambda_{0} \times 4 \lambda_{0}$, where $\lambda_{0}$ is a reference wavelength. Nonreflection boundary conditions are used to eliminate the disturbance caused by reflection. A circular plane source $\left(0.5 \lambda_{0}\right.$ in radius $)$ with a profile as $\exp [i(\omega t-l \phi)]$ is located on the $y$ - $z$ plane at $x=0$ with $\phi$ being the azimuthal angle. We conduct simulations for both $l=1$ [Fig. 1(a)] and $l=-1$ beams.

The simulated sound speed profile is $c=c_{0}(1-\alpha z)$, giving a refractive index,

$$
n=\frac{c_{0}}{c}=\frac{1}{1-\alpha z},
$$

and a reference wave-number $k_{0}=\omega / c_{0}=2 \pi / \lambda_{0}$. We start our simulation with the medium inhomogeneity $\alpha=0.2 \mathrm{~m}^{-1}$ and a reference wavelength $\lambda_{0}=1 \mathrm{~m}$. The refractive index 


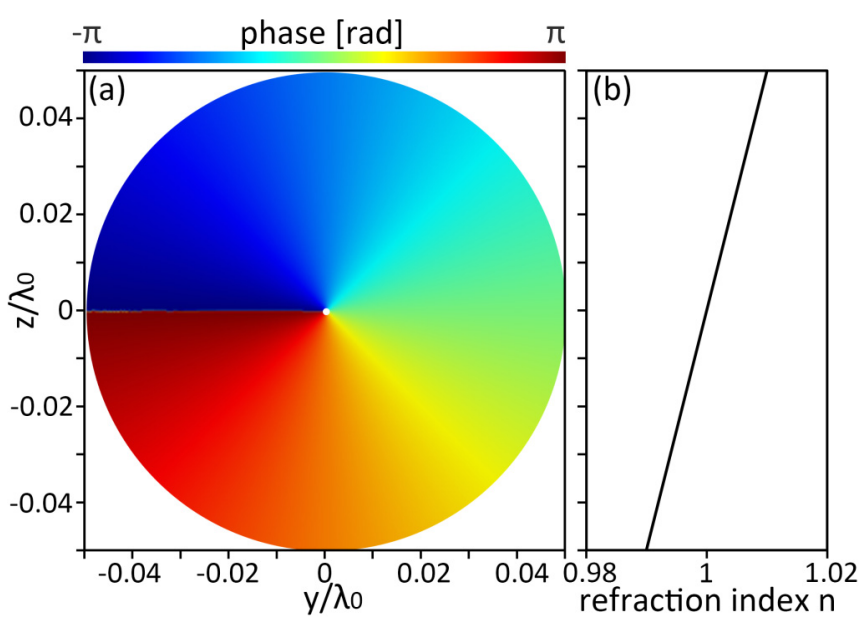

FIG. 1. (a) Simulated phase in the $y-z$ cross section at the initial source plane for a helicity $l=1$. (b) One example of the refraction index profile used in the simulation [see Eq. (1)].

profile $n$ is illustrated in Fig. 1(b). To study the dynamical change in the behaviors due to the variation of parameters, we conduct a series of simulations by varying the speed gradient $\alpha$ from 0.03 to 0.3 (fix the reference wave-number $k_{0}=2 \pi \mathrm{m}^{-1}$ ) and by varying the reference wave-number $k_{0}=2 \pi / \lambda_{0}$ from 5.0 to 8.3 (fix the gradient as $\alpha=0.1 \mathrm{~m}^{-1}$ ).

To precisely determine the location of the beam center during the propagation, we have reduced our mesh size near the singular core (a cylinder with radius of $0.05 \lambda_{0}$ ) to be as small as $1 / 200$ of the reference wavelength. This mesh size is around an order lower than the shift of the singular point location to be found in our simulations. The regions outside the cylinder have a regular mesh size of $1 / 16$ wavelength.

Figure 2(a) shows the phase in the $y-z$ cross section at the propagating distance $x=0.5 \lambda_{0}$ for the vortex beam with topological charges of $l=1$. The beam bends to the direction of sound speed descent $(+z$ direction) due to the refraction [Fig. 2(b)], which was recently simulated [51], whereas here we find that the beam center also has a transverse shift in the homogeneous $y$ direction. This transverse shift was not found in our prior simulations where the mesh size varies from $1 / 10$ to $1 / 5$ wavelength and the mesh near the core was not fine enough to identify the transverse shift.

The vortex beam with topological charge of $l=-1$ propagating in the same medium is shown in Fig. 2(c). We find that the transverse shift is of the same magnitude as the case of $l=$ 1 but in the opposite direction, revealing a dependence on the beam's helicity. We examine the transverse shifts as a function of the propagating distance for both $l= \pm 1$ [Fig. 2(d)]. We find that the transverse shift is linearly scaling with respect to the propagating distance whereas the shift direction depends on the sign of the helicity $l$.

Variations of the transverse shift with the medium inhomogeneity $\alpha$ and reference wave-number $k_{0}$ are shown in Figs. 3(a) and 3(b). The results show that, given a specific distance $x$, the transverse shift increases with speed gradient [Fig. 3(a)] and decreases with the wave number [Fig. 3(b)]. We determine the dependence by a linear fit and find that
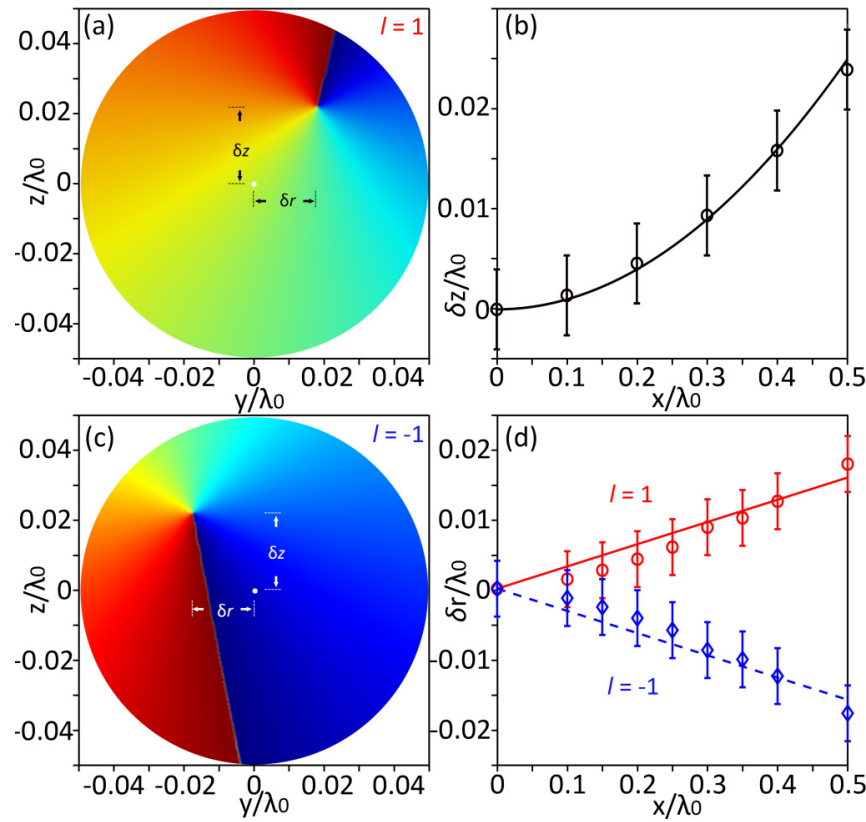

FIG. 2. (a) Simulated phase in the $y-z$ cross section at $x=0.5 \lambda_{0}$ for the vortex beam with a topological charge of $l=1$, showing the transverse Hall shift $\delta r$ in the homogeneous $y$ direction in addition to the refractive deflection $\delta z$ in the inhomogeneous $z$ direction where the corresponding shifts of the beam center (singular point) related to the original beam axis ( $x$ axis) are marked. (b) Vertical bending due to the refraction (black circles) with the solid line from a quadratic fit. (c) The same as (a) but for a beam of $l=-1$ where the transverse shift is of the same magnitude as (a) but in an opposite direction. (d) Transverse shift $\delta r$ increases with the propagating distance $x$ for both $l= \pm 1$ beams determined from the simulations and corresponding linear fits. For the vertical bending in (b), $l= \pm 1$ follows the same line. Error bars are determined from mesh size.

slope $\delta r / x$ of the fit is linearly scaling with $\alpha$ and with $1 / k_{0}$ [Fig. 3(c)], namely, $\delta r / x \propto \alpha / k_{0}$.

\section{MODELING OF THE ORBITAL HALL EFFECT IN ACOUSTICS}

We regard the helicity-dependent transverse shift observed here as the acoustic orbital Hall effect, which is the acoustic counterpart of the orbital Hall effect in optics. We reveal this effect by considering the equations of motion for the beam center in a smoothly inhomogeneous medium, which have been derived in the context of optics via the variation principle on the effective Lagrangian in the presence of Berry curvature $[2,4,9,10,15,52-54]$. Similar to the optical counterpart, the equations of wave-vector $\mathbf{k}$ and the displacement $\mathbf{r}$ of the acoustic vortex beam center as propagating in a smoothly inhomogeneous medium are as follows:

$$
\dot{\mathbf{k}}=k \nabla \ln n, \quad \text { and } \quad \dot{\mathbf{r}}=\frac{\mathbf{k}}{k}-l \frac{\mathbf{k} \times \mathbf{k}}{k^{3}},
$$

where the dot donates the derivative with respect to $s$, which is the length of a curved trajectory followed by the beam center. In the first equation, the gradient of the refractive index $(\nabla \ln n)$, representing the inhomogeneity of the medium, plays 


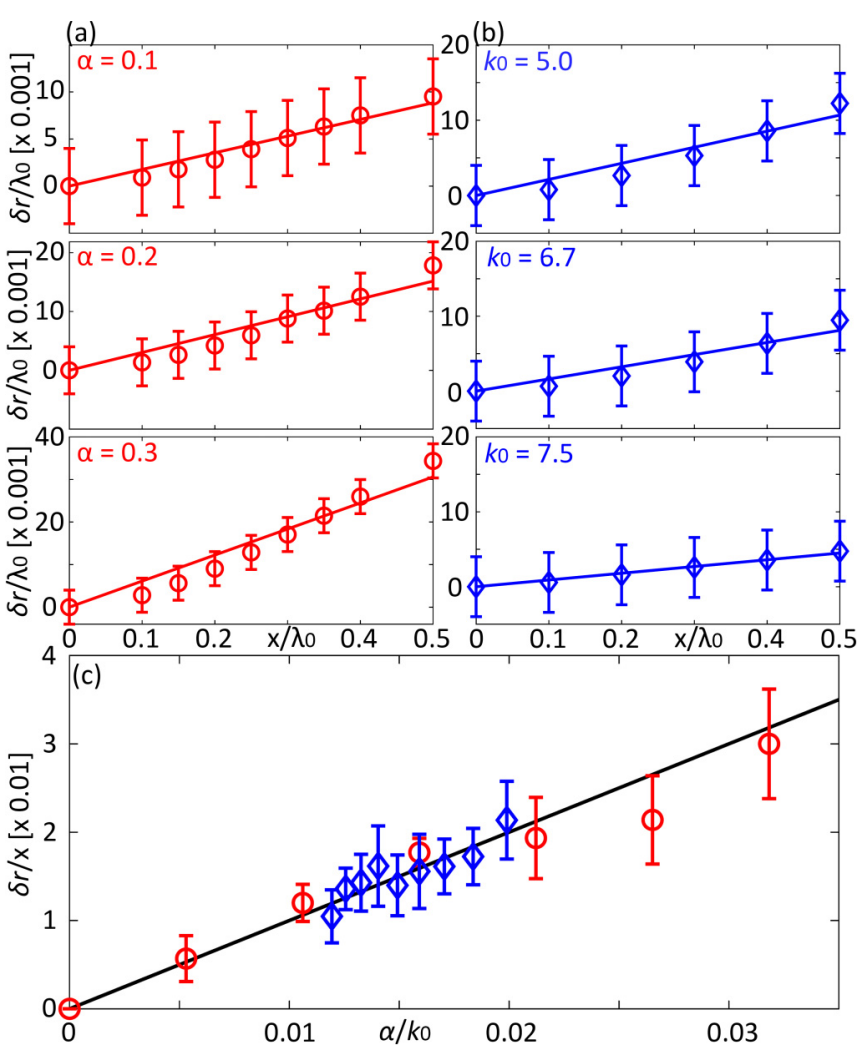

FIG. 3. Numerically simulated (circles/diamonds) versus linear fitted (solid lines) transverse Hall shifts as a function of propagating distance when (a) varying the gradient $\alpha$ (keeping $k_{0}=2 \pi \mathrm{m}^{-1}$ ) and (b) varying the wave-number $k_{0}$ (keeping $\alpha=0.1 \mathrm{~m}^{-1}$ ). (c) Derivative of transverse shift with respect to the propagating distance, i.e., $\delta r / x$ as a function of $\alpha / k_{0}$ determined from the simulated results in (a) and (b) [denoted by red circles and blue diamonds, respectively] and in comparison with theoretical prediction (solid line) from Eq. (6), i.e., $\delta r / x \propto \alpha / k_{0}$. Error bars in (a) and (b) come from the mesh size and in (c) come from the $95 \%$ confidence bounds of the linear fits.

the role of external force leading to the refraction of the beam in the inhomogenous direction. The first term in the second equation in (2) characterizes the ray followed by the beam center without considering the carried orbital angular momentum.

The second term in the second equation in (2), proportional to the orbital angular momentum $l$, is an addition to the vortex beam where the cross product of $\mathbf{k}$ and $\mathbf{k}$ leads to a transverse shift that is normal to both wave vector of the beam center and medium inhomogeneity. This phenomenon is, hence, regarded as the Hall effect since the shift is perpendicular to the "force," i.e., the medium inhomogeneity, in an analogy to the angular momentum Hall effect in optics [2]. The direction of the shift depends on the sign of the topological charge $l$ (Fig. 4). In our case, the sound speed gradient leads $\mathbf{k}$ to be in the $z$ direction, and $\mathbf{k}$ is dominantly along the $x$ direction. It follows from Eq. (2) that the shift is in the $y$ direction, consistent with our numerical simulations [Figs. 2(a) and 2(c)] and, consequently, validating our observation of the acoustic orbital Hall effect after decades of studies of acoustic vortex beams and orbital angular momenta.

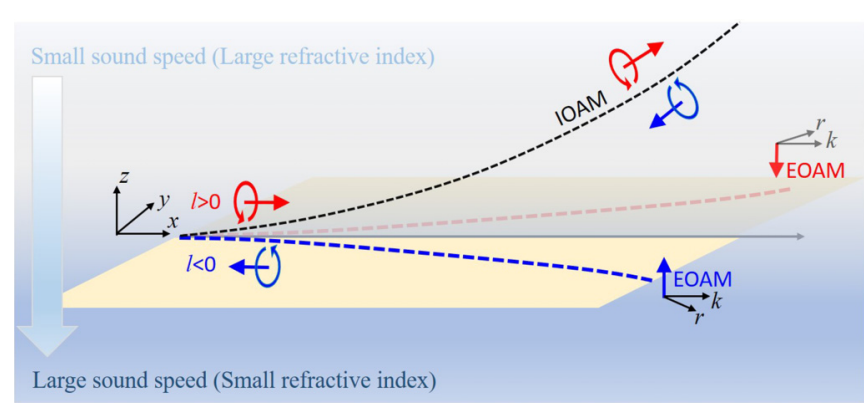

FIG. 4. Illustration of the orbital angular momentum Hall effect in acoustic vortex beam propagation in an inhomogeneous (gradient) medium, manifesting a helicity-dependent transverse shift in the homogeneous $y$ direction, accompanied by a coupling between extrinsic and intrinsic orbital angular momenta (denoted by EOAM and IOAM) and conservation of the total angular momenta along the gradient $z$ direction.

We now reveal the dependence of the shift on various parameters. Specifically, we consider a linearly stratified medium, whose refraction index as a function of depth was given in Eq. (1), and the first equation in (2) is simplified as

$$
\dot{\mathbf{k}}=k \frac{\alpha}{n} \widehat{\mathbf{z}}=k_{0} \frac{\alpha}{n^{2}} \hat{\mathbf{z}}
$$

Since the change in the wave vector is only along the $z$ direction, the expression of wave-vector $\mathbf{k}$ can be assigned as $\mathbf{k}=k_{x} \widehat{\mathbf{x}}+k_{z} \widehat{\mathbf{z}}$, where $k_{x}$ and $k_{z}$ are to be determined. Substituting $\mathbf{k}$ into Eq. (3), one has: (i) The wave number in the $x$ direction is constant and equal to the initial wave number, i.e., $k_{x}=k_{0}=\omega / c_{0}$, and (ii) the wave number in the $z$ direction can be solved from $d k_{z}=k_{0}\left(\alpha / n^{2}\right) d s$. Within the range considered in this paper, $\delta n \ll 1$ and zero-order approximation is applied, i.e., $d s \approx d x$ and $n \approx 1$. Hence, the wave vector is solved as

$$
\mathbf{k} \approx k_{0}[\widehat{\mathbf{x}}+\alpha x \widehat{\mathbf{z}}]
$$

The transverse shift in the homogeneous $y$ direction by the Hall effect can be solved by a contour integral along the ray of zero approximation,

$$
\delta \mathbf{r}=-l \int_{C}(\mathbf{k} \times d \mathbf{k}) / k^{3} .
$$

Combining Eqs. (3)-(5), we obtain

$$
\delta \mathbf{r}=\frac{l}{k_{0}} \alpha x \widehat{\mathbf{y}},
$$

which predicts that the shift is proportional to: (i) the helicity $l$, (ii) a typical magnitude of $k_{0}^{-1}$, (iii) the medium inhomogeneity gradient $\alpha$, and (iv) the propagating distance $x$. This theoretical prediction is compared with the numerical simulations in Fig. 3(c) where the derivative of transverse shift with respect to propagating distance $\delta r / x$ as a function of $\alpha / k_{0}$ is presented. The results confirm the agreement between the numerical observation and the theoretical prediction from Eq. (6), i.e., $\delta r / x=\alpha / k_{0}$ with the helicity $l=1$. 


\section{ORBIT-ORBIT INTERACTIONS AND ANGULAR MOMENTUM CONSERVATION}

The orbital angular momentum Hall shift represents the interactions between the intrinsic and the extrinsic orbital angular momentum with respect to the origin at $(x, y, z)=0$. The total angular momentum as a sum of extrinsic and intrinsic orbital angular momenta (with a proper normalization) can be expressed as

$$
\mathbf{J}=\mathbf{r} \times \mathbf{k}+l \mathbf{k} / k .
$$

The bending of the beam along the gradient direction by refraction immediately generates an extrinsic orbital angular momentum in the transverse direction (first term), which is $-(1 / 2) \alpha k_{0} x^{2} \hat{\mathbf{y}}$ in our case, following from the wave-vector $\mathbf{k}$ in Eq. (4) and the position vector $\mathbf{r}$ solved from the second equation in Eq. (2) in the zero approximation $(\dot{\mathbf{r}}=\mathbf{k} / k)$ to be

$$
\mathbf{r} \approx x \widehat{\mathbf{x}}+\frac{1}{2} \alpha x^{2} \widehat{\mathbf{z}}
$$

where the second term is the deflection in the inhomogeneous direction by refraction and is quadratic of propagating distance $x$ at the leading order [see Fig. 2(b)].

Besides, the wave refraction generates a variation of the intrinsic orbital angular momentum along the gradient direction [second term in Eq. (7)]. The variation is compensated by a variation of the extrinsic orbital angular momentum [first term in Eq. (7)] contributing from the transverse shift of the Hall effect, exhibiting the interactions of intrinsic and extrinsic angular momenta to guarantee the conservation of the total angular momenta. In our case, the variation in the $z$ component of the intrinsic angular momentum is

$$
\text { Intrinsic OAM variation }=\alpha l x \widehat{\mathbf{z}},
$$

and the extrinsic orbital angular momentum produced by the transverse shift is

$$
\text { Extrinsic OAM variation }=-\alpha l \widehat{x} \widehat{\mathbf{z}},
$$

manifesting the conservation of the total angular momenta along the gradient direction and the interactions between intrinsic and extrinsic orbital angular momenta for acoustic vortex beams in a smoothly inhomogeneous media.

Here the ray theory determines the motion of the beam center but not the field's intensity distribution. The intensity can be distorted to be transversely asymmetric with respect to the beam center. Such asymmetry was observed in our last simulation in Ref. [51] where it was explained by a superposition of vortex rotation and vertical refraction. The asymmetry perturbs the orbital angular momentum. This perturbation depends on the type of beam and is also affected by other factors, such as diffraction [20,55]. The further exploration of the asymmetry and the perturbation is beyond the scope of this present paper.

\section{SUGGESTED EXPERIMENTS}

The formula Eq. (6) assumes the range of refractive index variation is small. This is the case for many stratified media,

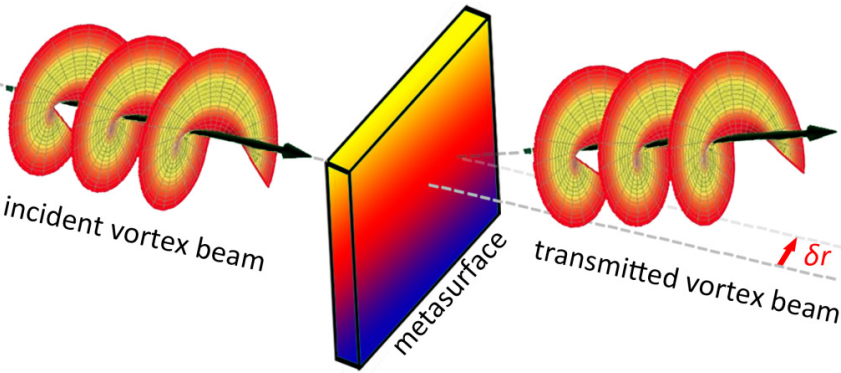

FIG. 5. Illustration of an experimental observation of the orbital Hall effect with the aid of an acoustic metasurface with a phase gradient. Transverse shift $\delta r$ can be observed from the transmitted field.

for example, the inhomogeneity of sound speed in ocean and atmospheric environments. To observe the transverse shift in a medium with a small variation, it would require a longdistance propagation. Take the ocean as an example where the range of sound speed variation is about $50 \mathrm{~m} / \mathrm{s}$ over a $4-\mathrm{km}$ depth, and the average sound speed is about $1500 \mathrm{~m} / \mathrm{s}$, implying an estimated shift of about only $2 \mathrm{~cm}$ for a $l=1$ sound vortex beam of $1-\mathrm{kHz}$ frequency traveling a distance of $10 \mathrm{~km}$ (horizontal range). The shift is very small even with such a long range of propagation. Such a weak shift could possibly be negligible for vortex-beam-based underwater alignment and communications [22,51,56-59]. A precise acoustic measurement to detect the slight shift would be difficult.

Experimental observation of a large shift by the acoustic orbital angular momentum Hall effect would be possible via generating a strongly inhomogeneous medium, which can be artificially created by the gradient of salinity or temperature. For example, one can create a sound speed gradient of about $0.38 \mathrm{~m} / \mathrm{s}$ per millimeter $\left(\alpha \approx 0.24 \mathrm{~m}^{-1}\right)$ in a laboratory tank setting by the salinity gradient [60]. By using sound waves in a frequency range of $1-10 \mathrm{kHz}$ in the fluid created in Ref. [60], one can effectively detect a transverse shift ranging from $6 \mathrm{~cm}$ to $6 \mathrm{~mm}$ at a distance of $1 \mathrm{~m}$ away from the sound source. The shift can be further amplified by a higher-order vortex beam source. A lower-frequency source would help to further enhance the shift.

In addition, a large transverse shift can be observed with the aid of acoustic metasurfaces possessing a spatial gradient. See the illustration in Fig. 5 where the metasurface has a spatial gradient along the vertical direction, whereas the vortex center has a shift along the transverse direction due to the orbital Hall effect. Such a gradient metasurface can be achieved by using a resonance-based structure [61,62] [Fig. 6(a)]. The structure is composed of individual elements with each having a hybrid structure formed by coupling a straight tube with a series connection of four cavities acting as acoustic resonators [see Fig. 6(b) for the cross section of one element]. The element can effectively adjust the phase of the transmitted waves by varying the geometry parameter $d / D$ whereas keeping a high transmission efficiency larger than $80 \%$ for airborne sound [see the simulated results in Fig. 6(c)]. As an example, 16 elements with different $d / D$ 's are selected to eventually build a metasurface with a phase gradient of 

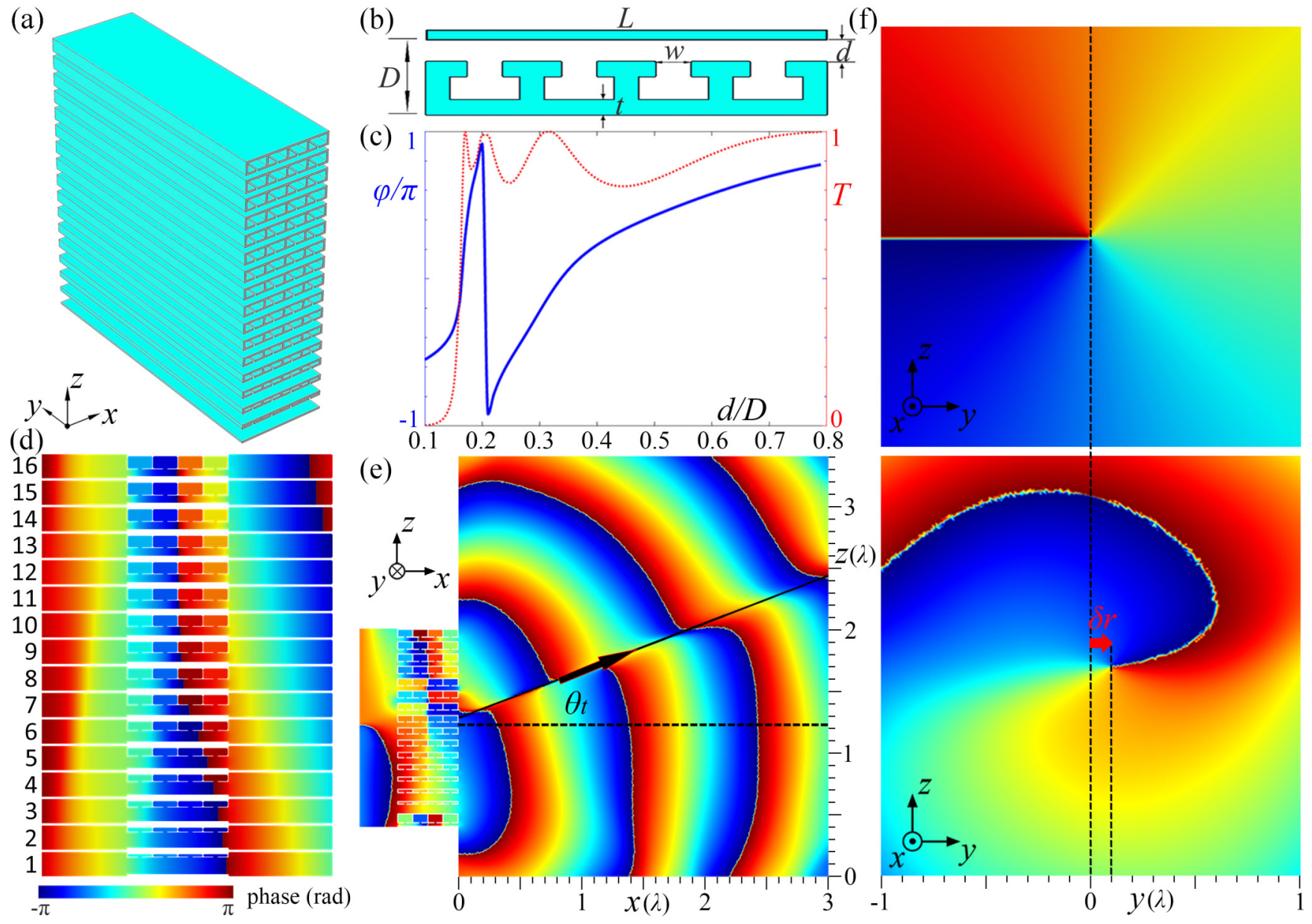

FIG. 6. (a) Illustration of the metasurface with the cross section of one element shown in (b). Parameters are chosen as $L=0.5 \lambda, D=$ $0.1 \lambda, t=0.01 \lambda$, and $w=0.015 \lambda$ with $\lambda$ being the wavelength. (c) Simulated phase shift covering the range of $2 \pi$ can be effectively adjusted by varying the parameter $d / D$ with the transmission efficiency larger than $80 \%\left(T=\left|p_{t} / p_{i}\right|\right.$ with $p_{i}$ and $p_{t}$ being the incident and transmitted fields). (d) Simulated phases of the 16 elements used in the simulation, giving the phase gradient of $0.8 \pi$ per wavelength. (e) Phase on the $x-z$ cross section at $y=0$ where the wave vector of the transmitted beam follows along the black arrow with an angle $\theta_{t}$ to the normal line (dashed black line). (f) Phase profile of the incident wave (top panel) and the transmitted wave (bottom panel) on the $y$ - $z$ cross section where the transverse shift is marked between the two black dashed lines.

$0.8 \pi$ per wavelength; see Fig. 6(d). The metasurface can be printed using three-dimensional printing technology.

A phase on the $x-z$ cross section at $y=0$ for the propagation of a vortex beam by simulations using the proposed structure is shown in Fig. 6(e) where the wave vector of the transmitted beam follows a refracted angle $\theta_{t}$ given by the generalized Snell's law [63],

$$
\theta_{t}=\sin ^{-1}\left(\frac{1}{k} \frac{d \phi}{d z}\right),
$$

where $d \phi / d z$ represents the spatial phase gradient of the metasurface along the gradient $z$ direction, which is $0.8 \pi$ per wavelength in our example, giving that $\theta_{t}=23.6^{\circ}$. The acoustic orbital Hall effect can be observed by measuring the transverse shift of the vortex center in the $y$ direction, which is about $0.1 \lambda$ difference between the incident and refracted vortex beams, as shown by the two black dashed lines in Fig. 6(f). The incident vortex beam used here can be generated, for example, by four individual sources with the same amplitude and linearly increasing phases $(0, \pi / 2, \pi, 3 \pi / 2)$.
The experiment proposed here allows for the observation of the transverse shifts in air. A large phase gradient helps to amplify the shift. More complex beams, media, and structures could also be used.

\section{CONCLUSION AND REMARKS}

To conclude, the orbital angular momentum Hall effect in acoustics is observed via numerical simulations. A vortex beam carrying the orbital angular momentum propagating in a smoothly inhomogeneous medium is considered. The transverse shifts related to the orbital angular momentum Hall effect are predicted, observed, and then characterized by a ray formula in the inhomogeneity approximation. These results demonstrate that the orbital angular momentum of sound can also have interesting topological properties that may find applications in the manipulations of sound signals. Our paper opens up the fundamental study of the acoustic Hall effect and orbital angular momentum in an inhomogeneous medium and inspires relevant applications in the future. Note that the 
shift is proportional to sound speed, but the sound speed is several orders smaller than optical waves. The experiments we proposed herein using metasurfaces or stratified fluids would allow the observation of a large shift.

\section{ACKNOWLEDGMENT}

We acknowledge the discussion with Dr. Z. Zou. The research was partially supported by the start-up fund from the University of Mississippi.
[1] V. S. Liberman and B. Y. Zel'dovich, Spin-orbit interaction of a photon in an inhomogeneous medium, Phys. Rev. A 46, 5199 (1992).

[2] M. Onoda, S. Murakami, and N. Nagaosa, Hall Effect of Light, Phys. Rev. Lett. 93, 083901 (2004).

[3] A. Kavokin, G. Malpuech, and M. Glazov, Optical Spin Hall Effect, Phys. Rev. Lett. 95, 136601 (2005).

[4] K. Y. Bliokh, Geometrical Optics of Beams with Vortices: Berry Phase and Orbital Angular Momentum Hall Effect, Phys. Rev. Lett. 97, 043901 (2006).

[5] X. Yin, Z. Ye, J. Rho, Y. Wang, and X. Zhang, Photonic spin Hall effect at metasurfaces, Science 339, 1405 (2013).

[6] K. Y. Bliokh, D. Smirnova, and F. Nori, Quantum spin Hall effect of light, Science 348, 1448 (2015).

[7] S. Fu, C. Guo, G. Liu, Y. Li, H. Yin, Z. Li, and Z. Chen, SpinOrbit Optical Hall Effect, Phys. Rev. Lett. 123, 243904 (2019).

[8] J. E. Hirsch, Spin Hall Effect, Phys. Rev. Lett. 83, 1834 (1999).

[9] S. Zhang, S. Murakami, N. Nagaosa, X. Qi, and Y. Wu, The spin Hall effect, Science 301, 1348 (2003).

[10] J. Sinova, D. Culcer, Q. Niu, N. A. Sinitsyn, T. Jungwirth, and A. H. MacDonald, Universal Intrinsic Spin Hall Effect, Phys. Rev. Lett. 92, 126603 (2004).

[11] X. Ling, X. Zhou, K. Huang, Y. Liu, C.-W. Qiu, H. Luo, and S. Wen, Recent advances in the spin Hall effect of light, Rep. Prog. Phys. 80, 066401 (2017).

[12] S. Xiao, J. Wang, F. Liu, S. Zhang, X. Yin, and J. Li, Spindependent optics with metasurfaces, Nanophotonics 6, 215 (2017).

[13] Y. Liu, Y. Ke, H. Luo, and S. Wen, Photonic spin Hall effect in metasurfaces: A brief review, Nanophotonics 6, 51 (2017).

[14] O. C. de Beauregard and C. Imbert, Quantized Longitudinal and Transverse Shifts Associated with Total Internal Reflection, Phys. Rev. Lett. 28, 1211 (1972).

[15] S. I. Vinitskii, V. L. Derbov, V. M. Dubovik, B. L. Markovski, and Y. P. Stepanovskii, Topological phases in quantum mechanics and polarization optics, Sov. Phys. Usp. 33, 403 (1990).

[16] K. Y. Bliokh and Y. P. Bliokh, Conservation of Angular Momentum, Transverse Shift, and Spin Hall Effect in Reflection and Refraction of an Electromagnetic Wave Packet, Phys. Rev. Lett. 96, 073903 (2006).

[17] V. G. Fedoseyev, Spin-independent transverse shift of the centre of gravity of a reflected and of a refracted light beam, Opt. Commun. 193, 9 (2001).

[18] R. Dasgupta and P. K. Gupta, Experimental observation of spin-independent transverse shift of the centre of gravity of a reflected laguerre-gaussian light beam, Opt. Commun. 257, 91 (2006).

[19] V. G. Fedoseyev, Transformation of the orbital angular momentum at the reflection and transmission of a light beam on a plane interface, J. Phys. A: Math. Theor. 41, 505202 (2008).
[20] E. Nordblad, Transverse and lateral shifts of the center of gravity of a refracted nonparaxial bessel beam, Phys. Rev. A 85, 013847 (2012).

[21] C. M. Cisowski and R. R. B. Correia, Splitting an optical vortex beam to study photonic orbit-orbit interactions, Opt. Lett. $\mathbf{4 3}$ 499 (2018).

[22] B. T. Hefner and P. L. Marston, An acoustical helicoidal wave transducer with applications for the alignment of ultrasonic and underwater systems, J. Acoust. Soc. Am. 106, 3313 (1999).

[23] L. Zhang and P. L. Marston, Angular momentum flux of nonparaxial acoustic vortex beams and torques on axisymmetric objects, Phys. Rev. E 84, 065601(R) (2011).

[24] K. Volke-Sepúlveda, A. O. Santillán, and R. R. Boullosa, Transfer of Angular Momentum to Matter from Acoustical Vortices in Free Space, Phys. Rev. Lett. 100, 024302 (2008).

[25] J.-L. Thomas, T. Brunet, and F. çois Coulouvrat, Generalization of helicoidal beams for short pulses, Phys. Rev. E 81, 016601 (2010).

[26] A. Anhäuser, R. Wunenburger, and E. Brasselet, Acoustic Rotational Manipulation Using Orbital Angular Momentum Transfer, Phys. Rev. Lett. 109, 034301 (2012).

[27] ZhenYu Hong, J. Zhang, and B. W. Drinkwater, Observation of Orbital Angular Momentum Transfer from Bessel-Shaped Acoustic Vortices to Diphasic Liquid-Microparticle Mixtures, Phys. Rev. Lett. 114, 214301 (2015).

[28] X. Jiang, Y. Li, B. Liang, Jian-chun Cheng, and L. Zhang, Convert Acoustic Resonances to Orbital Angular Momentum, Phys. Rev. Lett. 117, 034301 (2016).

[29] C. Shi, M. Dubois, Y. Wang, and X. Zhang, High-speed acoustic communication by multiplexing orbital angular momentum, Proc. Natl. Acad. Sci. USA 114, 7250 (2017).

[30] K. Y. Bliokh and F. Nori, Spin and orbital angular momenta of acoustic beams, Phys. Rev. B 99, 174310 (2019).

[31] Z. Yang, F. Gao, X. Shi, X. Lin, Z. Gao, Y. Chong, and B. Zhang, Topological Acoustics, Phys. Rev. Lett. 114, 114301 (2015).

[32] M. Xiao, W.-J. Chen, W.-Y. He, and C. T. Chan, Synthetic gauge flux and Weyl points in acoustic systems, Nat. Phys. 11, 920 (2015).

[33] R. Fleury, A. B. Khanikaev, and A. Alu, Floquet topological insulators for sound, Nat. Commun. 7, 1 (2016).

[34] C. He, X. Ni, H. Ge, X.-C. Sun, Y.-B. Chen, M.-H. Lu, X.-P. Liu, and Y.-F. Chen, Acoustic topological insulator and robust one-way sound transport, Nat. Phys. 12, 1124 (2016).

[35] J. Mei, Z. Chen, and Y. Wu, Pseudo-time-reversal symmetry and topological edge states in two-dimensional acoustic crystals, Sci. Rep. 6, 1 (2016).

[36] Y.-G. Peng, C.-Z. Qin, D.-G. Zhao, Y.-X. Shen, X.-Y. Xu, M. Bao, H. Jia, and X.-F. Zhu, Experimental demonstration of anomalous floquet topological insulator for sound, Nat. Commun. 7, 13368 (2016). 
[37] Z. Zhang, Q. Wei, Y. Cheng, T. Zhang, D. Wu, and X. Liu, Topological Creation of Acoustic Pseudospin Multipoles in a Flow-Free Symmetry-Broken Metamaterial Lattice, Phys. Rev. Lett. 118, 084303 (2017).

[38] Y. Deng, H. Ge, Y. Tian, M. Lu, and Y. Jing, Observation of zone folding induced acoustic topological insulators and the role of spin-mixing defects, Phys. Rev. B 96, 184305 (2017).

[39] H. He, C. Qiu, L. Ye, X. Cai, X. Fan, M. Ke, F. Zhang, and Z. Liu, Topological negative refraction of surface acoustic waves in a weyl phononic crystal, Nature (London) 560, 61 (2018).

[40] Z. Zhang, Y. Tian, Y. Wang, S. Gao, Y. Cheng, X. Liu, and J. Christensen, Directional acoustic antennas based on valley-Hall topological insulators, Adv. Mater. 30, 1803229 (2018).

[41] X. Zhang, M. Xiao, Y. Cheng, M.-H. Lu, and J. Christensen, Topological sound, Commun. Phys. 1, 97 (2018).

[42] W. Zhu, X. Fang, D. Li, Y. Sun, Y. Li, Y. Jing, and H. Chen, Simultaneous Observation of a Topological Edge State and Exceptional Point in an Open and Non-Hermitian Acoustic System, Phys. Rev. Lett. 121, 124501 (2018).

[43] J. Lu, C. Qiu, W. Deng, X. Huang, F. Li, F. Zhang, S. Chen, and Z. Liu, Valley Topological Phases in Bilayer Sonic Crystals, Phys. Rev. Lett. 120, 116802 (2018).

[44] M. A. Bandres, S. Wittek, G. Harari, M. Parto, J. Ren, M. Segev, D. N. Christodoulides, and M. Khajavikhan, Topological insulator laser: Experiments, Science 359, eaar4005 (2018).

[45] G. Ma, M. Xiao, and C. T. Chan, Topological phases in acoustic and mechanical systems, Nat. Rev. Phys. 1, 281 (2019).

[46] X. Ni, M. Weiner, A. Alù, and A. B. Khanikaev, Observation of higher-order topological acoustic states protected by generalized chiral symmetry, Nature Mater. 18, 113 (2019).

[47] H. Xue, Y. Yang, F. Gao, Y. Chong, and B. Zhang, Acoustic higher-order topological insulator on a kagome lattice, Nature Mater. 18, 108 (2019).

[48] Y. Ding, Y. Peng, Y. Zhu, X. Fan, J. Yang, B. Liang, X. Zhu, X. Wan, and J. Cheng, Experimental Demonstration of Acoustic Chern Insulators, Phys. Rev. Lett. 122, 014302 (2019).

[49] Y. Long, J. Ren, and H. Chen, Unsupervised Manifold Clustering of Topological Phononics, Phys. Rev. Lett. 124, 185501 (2020).

[50] S. Wang, G. Ma, and C. T. Chan, Topological transport of sound mediated by spin-redirection geometric phase, Sci. Adv. 4, eaaq1475 (2018).
[51] X.-D. Fan, Z. Zou, and L. Zhang, Acoustic vortices in inhomogeneous media, Phys. Rev. Research 1, 032014(R) (2019).

[52] J. Yi, Physics from topology and structures, in Topology in Condensed Matter, edited by M. I. Monastyrsky (Springer, Berlin/Heidelberg, 2006), pp. 117-137.

[53] K. Y. Bliokh and Y. P. Bliokh, Modified geometrical optics of a smoothly inhomogeneous isotropic medium: The anisotropy, Berry phase, and the optical magnus effect, Phys. Rev. E 70, 026605 (2004).

[54] K. Y. Bliokh and Y. P. Bliokh, Topological spin transport of photons: The optical Magnus effect and Berry phase, Phys. Lett. A 333, 181 (2004).

[55] K. Y. Bliokh and A. S. Desyatnikov, Spin and orbital Hall effects for diffracting optical beams in gradient-index media, Phys. Rev. A 79, 011807(R) (2009).

[56] X. Jiang, C. Shi, Y. Wang, J. Smalley, J. Cheng, and X. Zhang, Nonresonant Metasurface for Fast Decoding in Acoustic Communications, Phys. Rev. Appl. 13, 014014 (2020).

[57] V. Bollen and P. L. Marston, Phase and amplitude evolution of backscattering by a sphere scanned through an acoustic vortex beam: Measured helicity projections, J. Acoust. Soc. Am. 148, EL135 (2020).

[58] H. Zhou, J. Li, C. Gong, K. Guo, and Z. Guo, Measuring the topological charges of acoustic vortices by apertures, J. Acoust. Soc. Am. 148, 167 (2020).

[59] X. Li, Y. Li, Q. Ma, G. Guo, J. Tu, and D. Zhang, Principle and performance of orbital angular momentum communication of acoustic vortex beams based on single-ring transceiver arrays, J. Appl. Phys. 127, 124902 (2020)

[60] L. K. Zhang and H. L. Swinney, Sound propagation in a continuously stratified laboratory ocean model, J. Acoust. Soc. Am. 141, 3186 (2017).

[61] Y. Li, X. Jiang, B. Liang, J.-c. Cheng, and L. Zhang, Metascreen-Based Acoustic Passive Phased Array, Phys. Rev. Appl. 4, 024003 (2015).

[62] X. Jiang, Y. Li, and L. Zhang, Thermoviscous effects on sound transmission through a metasurface of hybrid resonances, J. Acoust. Soc. Am. 141, EL363 (2017).

[63] N. Yu, P. Genevet, M. A. Kats, F. Aieta, J.-P. Tetienne, F. Capasso, and Z. Gaburro, Light propagation with phase discontinuities: Generalized laws of reflection and refraction, Science 334, 333 (2011). 